\title{
OBSERVATION OF STRUCTURE IN ELASTIC PP SCATTERING AT 100 AND $200 \mathrm{GeV} / c^{\text {th }}$
}

\author{
C.W. AKERLOF, R. KOTTHAUS*, R.L. LOVELESS, D.I. MEYER \\ Physics Department University of Michigan, Ann Arbor, Michigan 48104, USA \\ I. AMBA TS, W.T. MEYER ${ }^{* *}$, C.E.W. WARD \\ Argonne National Laboratory, Argonne, Ilinois 60439, USA \\ D.P. EARLTY, R.A. LUNDY, S.M. PRUSS, D.D. YOVANOVITCH \\ Fermi National Accelerator Laboratory, Batavia, Ilinois 60510, USA \\ D.R. RUST \\ Physics Department, University of Indiana, Bloomington, Indiana 47401, USA
}

Revised manuscript received 28 September 1975

\begin{abstract}
The elastic differential cross section for pp scattering has been measured up to a momentum transfer of ${ }_{;} t \mid=3(\mathrm{GeV} / c)^{2}$ at $100 \mathrm{GeV} / c$ and $200 \mathrm{GeV} / c$ incident momenta. The $200 \mathrm{GeV} / c$ measurements shows a diffractive like dip at $|t|=1.5 \mathrm{GeV} / c$ while no such dip is seen in the $100 \mathrm{GeV} / c$ data.
\end{abstract}

Several measurements of elastic pp scattering at ISR energies $[1,2]$ in the momentum transfer region, $|t|>1.0(\mathrm{GeV} / c)^{2}$ exhibit a pronounced dip structure in the differential cross section $\mathrm{d} \sigma / \mathrm{d} t$ at $|t| \approx 1.4$ $(\mathrm{GeV} / c)^{2}$ often attributed to diffractive effects. These measurements span a wide range of center-of-mass energies: $23.5-53 \mathrm{GeV}$ corresponding to incident laboratory momenta from $300 \mathrm{GeV} / c$ to $2100 \mathrm{GeV} / c$. Similar structure has not been observed at laboratory momenta of $30 \mathrm{GeV} / c$ and below, although there is a break in the cross section near this $t$ value [3].

We have measured pp elastic scattering at 100 and $200 \mathrm{GeV} / c$ incident momentum to examine how this structure develops as a function of energy.

The experiment was performed in the M1E beam of the Meson Area at the Fermi National Accelerator Laboratory. The experimental apparatus shown in fig. 1 consists of a hydrogen target, a forward spectrometer utilized two bending magnets of $20 \times 60 \mathrm{~cm}$

\footnotetext{
Work supported by the U.S. E.R.D.A.

* Present address: Max-Planck-Institut für Physik und Astrophysik, München, W. Germany.

** Present address: Stanford Linear Accelerator Center, Stanford, California.
}

aperture, each $183 \mathrm{~cm}$ long, giving a total $B d l$ of $70 \mathrm{kG}$ meters. Particle track positions were measured with magnetostrictive wire spark chambers arranged in modules of 4 gaps each. Two chambers were aligned to provide $(x, y)$ coordinates and two measured $(u, v)$ coordinates rotated by $30^{\circ}$ with respect to the vertical. Three such modules were used in the forward spectrometer; two placed immediately fore and aft of the bending magnets and the third 18.3 meters downstream of the bend center. The momentum resolution of this forward arm was measured to be $\Delta p / p= \pm 1 \%$ at $200 \mathrm{GeV} / c$. The fourth module of spark chambers, containing 6 gaps, $4(x, y)$ and $2(u, v)$ type, were used as the recoil detector. Two scintillator hodoscopes of 10 elements in both the horizontal and vertical plane defined the direction of an incident particle. The first hodoscope (not shown in fig. 1) was $30 \mathrm{~m}$ upstream from the hydrogen target. The hodoscopes with a spatial resolution of $\pm 1.5 \mathrm{~mm}$ determined incident particle directions to a precision of $\pm 0.07 \mathrm{mrad}$. The beam was operated with a momentum definition of $\Delta p / p \approx \pm 0.5 \%$ and at an intensity of about $10^{6}$ particles per $0.8 \mathrm{sec}$ long accelerator spill.

The spark chamber trigger required a beam particle, 


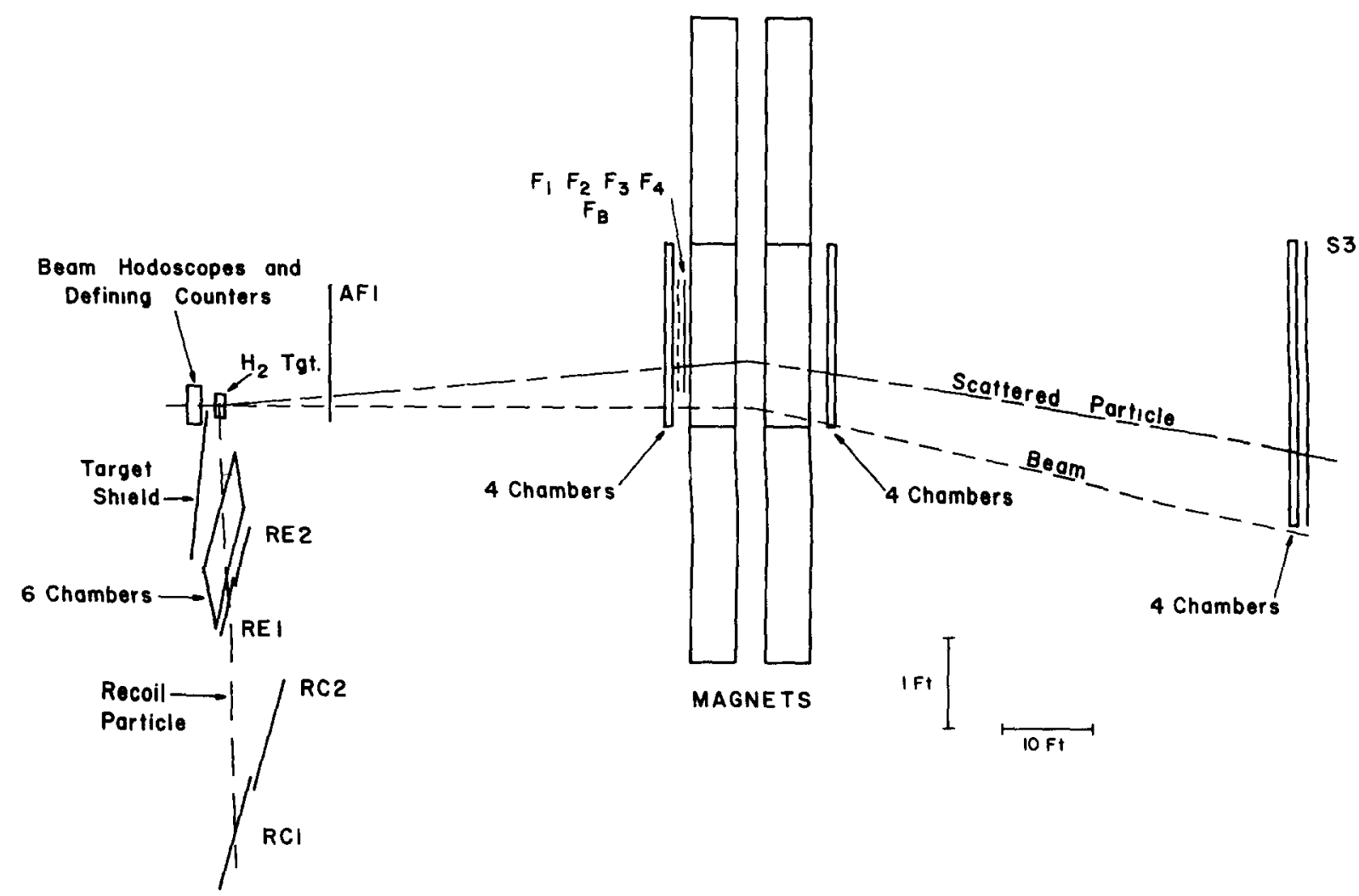

Fig. 1. The experimental set-up. Note 10:1 foreshortening along the incident beam direction.

previously labeled by two gas differential Cerenkov counters to be a proton, no signal from a veto counter containing a $2.5 \times 2.5 \mathrm{~cm}$ hole upstream of the hydrogen counter, and finally signals from scintillators in both the forward and recoil arms. Rather important in rejecting inelastic events was a veto counter (AFI) behind two radiation lengths of $\mathrm{Pb} 2.5 \mathrm{~m}$ downstream of the hydrogen target. A hole in this counter allowed all elastic events to be accepted which were within the magnet aperture while a large fraction of inelastic events were rejected.

The acceptance of this system is mostly determined by the geometry of the recoil arm and is quite uniform for $0.1 \leqslant|t| \leqslant 7(\mathrm{GeV} / c)^{2}$. Below a $|t|$ value of 0.1 $(\mathrm{GeV} / c)^{2}$ the short range of the recoil protons and the dimensions of the hydrogen target $(4 \mathrm{~cm}$ diameter and $30 \mathrm{~cm}$ long) reduce the effective acceptance quite rapidly. During some of the data taking the trigger counters $F_{1}$ and $F_{2}$ were disconnected to suppress triggers with a $|t|$ value $\leqslant 0.6(\mathrm{GeV} / c)^{2}$ which were abundant compared to the total trigger rate with $|t| \geqslant 0.6(\mathrm{GeV} / c)^{2}$

The trigger defined above selected approximately equal numbers of elastic and inelastic events at small $|t|$. This ratio became of the order in a hundred at large values of $|t|$. These inelastic events were eliminated by applying appropriate cuts to the momentum of the forward particle, the coplanarity, and the included angle of the forward and recoil particles.

Fig. 2 is a scatter plot of events in the opening and coplanarity angles plane. Included in the plot are all events which passed a missing mass cut centered at the proton mass with $\Delta M^{2}= \pm 10(\mathrm{GeV})^{2}$ and with a $|t|$ value larger than $0.8(\mathrm{GeV} / \mathrm{c})^{2}$. The projections of this scatter plot in both opening angle and coplanarity exhibit elastic peaks with $0.6 \mathrm{mrad}$ (coplanarity) and $0.4 \mathrm{mrad}$ (opening angle) HWHM. These two angular cuts define colinearity in the C.M. frame which corresponds to a momentum imbalance of less than \pm 75 $\mathrm{MeV}$ at $200 \mathrm{GeV} / c$. The tails on both sides of the peaks are taken as inelastic background, assumed to be flat, and subtracted from the signal. The relative amount of 

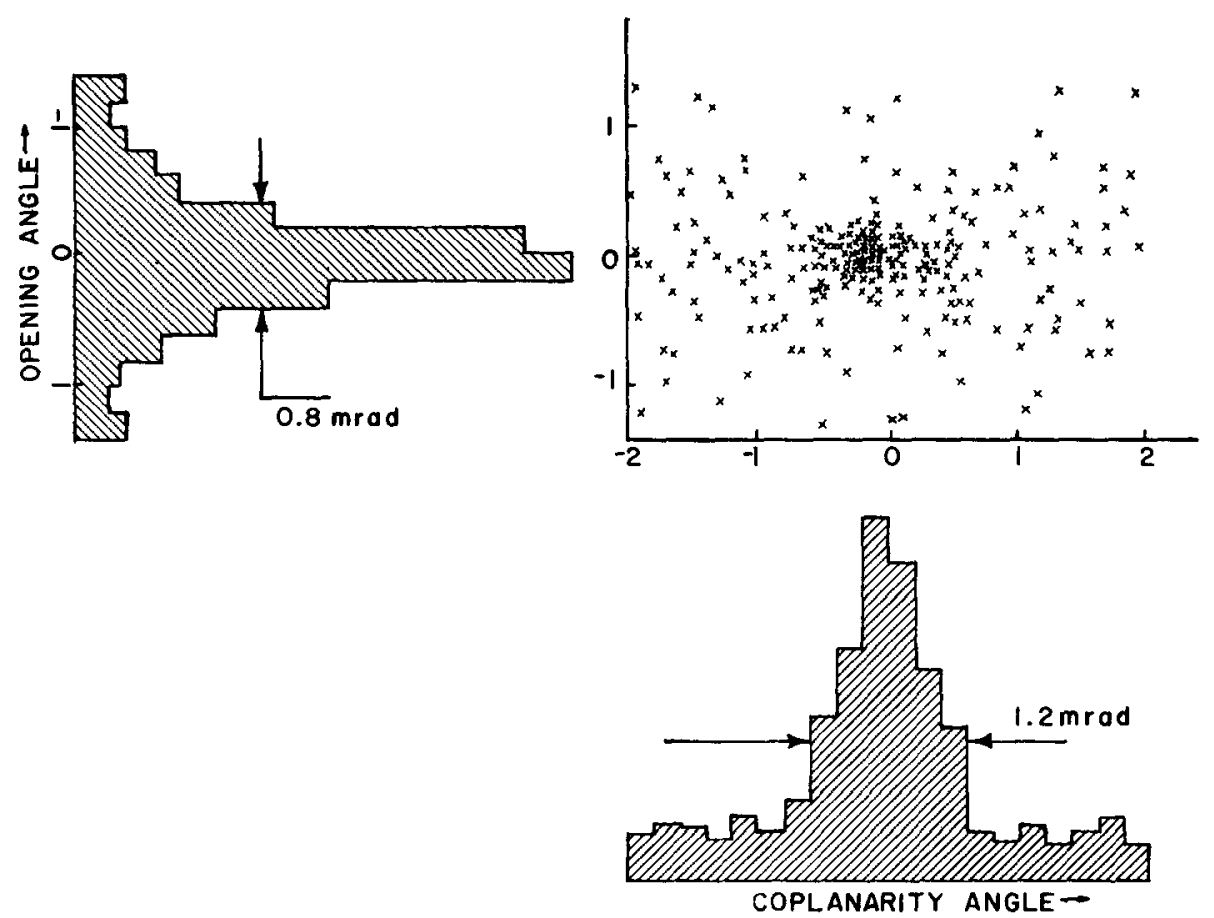

Fig. 2. Scatter plot of events in opening angle-coplanarity plane at $200 \mathrm{GeV} / \mathrm{c}$ incident momentum. Included are the events with $|t|>0.8(\mathrm{GeV} / c)^{2}$. The projected distribution on either opening angle or coplanarity are also shown.

this background increases as a function of $t$ and was $10 \%$ of the signal at $|t|=1(\mathrm{GeV} / c)^{2}$.

Fig. 3 shows the opening angle and coplanarity angle distributions with no background subtraction as a function of $t$ in the dip region for $200 \mathrm{GeV} / c$ incident momentum. The disappearance of the elastic peak from both coplanarity and opening angle distributions in the dip region, $1.35<|t|<1.45(\mathrm{GeV} / \mathrm{c})^{2}$, and its return at higher $|t|$ values is apparent. This behavior was not observed in the $100 \mathrm{GeV} / c$ data, where the signal remains visible throughout the same range of $|t|$. Therefore, we conclude that the onset of the minimum in the elastic $\mathrm{pp}$ scattering occurs between 100 and $200 \mathrm{GeV} / c$ incident proton momentum.

The $|t|$ resolution in the dip region was $\Delta t=0.025$ $(\mathrm{GeV} / c)^{2}$ at $200 \mathrm{GeV} / c$ and somewhat better for $100 \mathrm{GeV} / c$ incident momentum. In both cases a limited number of pion events were measured simultaneously - their $|t|$ distribution does not show any similar structure. We can thus rule out spurious effects such as non-uniform spark chamber efficiency, scintillators, etc. as the cause of the observed structure.
The data corrected with a smooth background extrapolation and subtraction under the observed elastic peaks and cut with $\pm 0.5 \mathrm{mr}$ and $\pm 0.8 \mathrm{mr}$ cuts in the opening angle and coplanarity angle is shown in fig. 4. The overall normalization was obtained from the small angle elastic scattering data taken in the same apparatus during a separate run with $F_{1}, F_{2}$, $F_{3}$, and $F_{4}$ active.

The accuracy of the small $|t|$ data normalization is $\pm 7 \%$; however, we have increased the normalization uncertainty to $\pm 20 \%$ to allow for possible variations in system efficiency at high beam rates.

The data also shows indications of a slight bump around $-t=0.8(\mathrm{GeV} / c)^{2}$ at both 100 and $200 \mathrm{GeV} / c$.

The Chou and Yang [4] model successfully predicted the dip in the elastic pp scattering assuming that the hadronic distribution reflects the charge distribution within the proton. This model, and additional refinements of it [5], predict the dip to occur at $-t \approx 1.4(\mathrm{GeV} / c)^{2}$ using the same parameterization of the E.M. form factors as measured in electron proton scattering. This diffractive like dip is well 


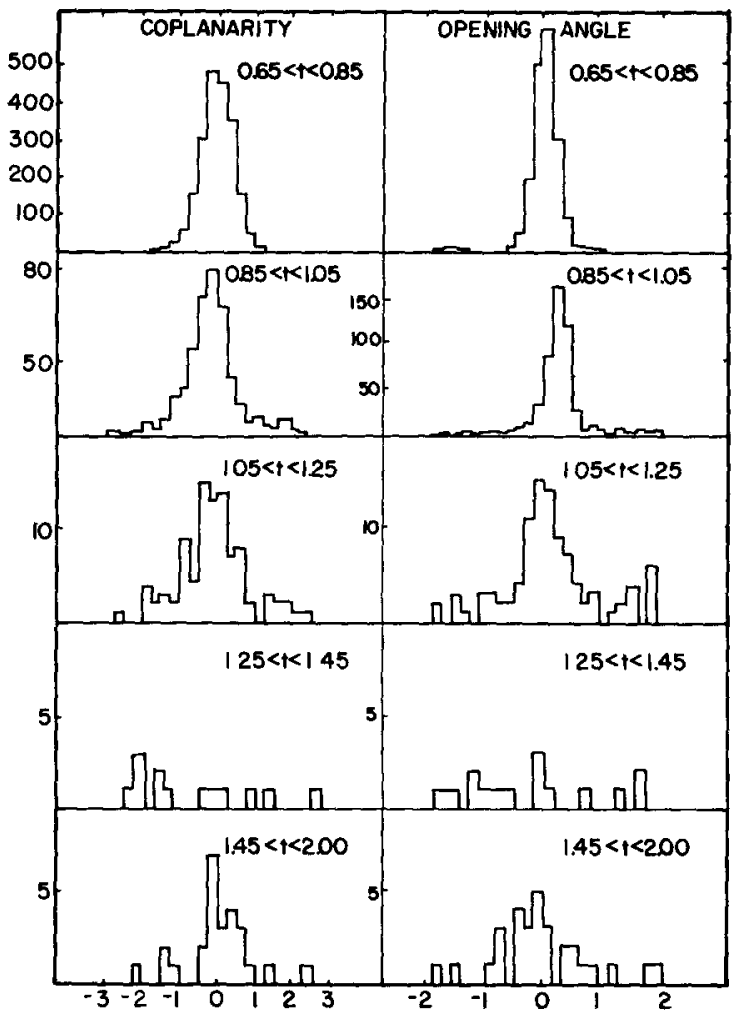

Fig. 3. Opening angle and coplanarity distributions as a function of momentum transfer $|t|$ at $200 \mathrm{GeV} / c$ incident momentum.

established at all ISR energies.

Our measurements indicate that the dip develops only above $100 \mathrm{GeV} / c$ incident momentum. There is a parameterization by Phillips and Barger [6] which predicts the $s$-dependence of the dip structure. Our data does not contradict that parameterization but is not extensive enough to confirm its major features. A simple interpretation would be that in the absence of a real part in the scattering amplitude, a diffraction dip in the imaginary part is observable at high energies. The filling of the dip structure at lower energies is then due to the real part of the scattering amplitude, which would be expected to be larger at lower energies. The measurements of the $\rho$ parameter, $\rho=\operatorname{Re} f(s) / \operatorname{Im}$ $f(s)$ by Bartenev et al. [7] show that, at least near $0^{\circ}$, $\rho=0$ at $200 \mathrm{GeV} / c$ incident momentum.

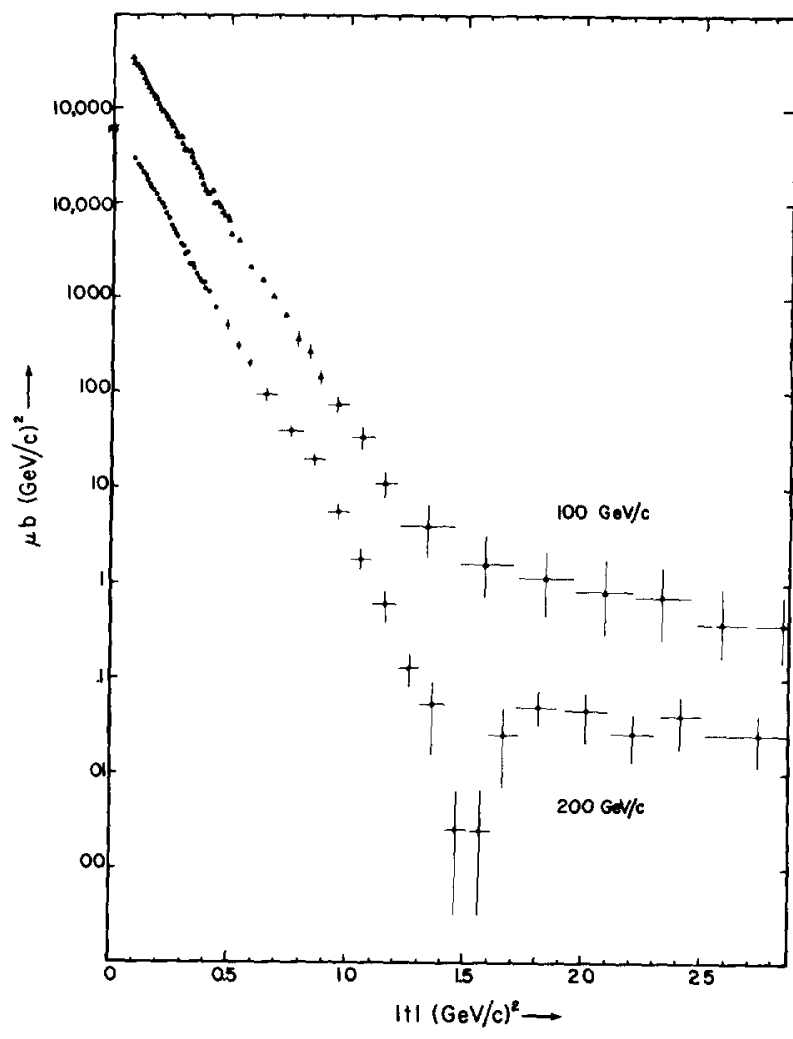

Fig. 4. Differential cross sections $\mathrm{d} \sigma / \mathrm{d} t$ for 100 and $200 \mathrm{GeV} / c$ incident momenta.

We wish to thank the Fermilab Accelerator staff for the good performance of the accelerator during our runs and to Dr. P. Koehler and the Meson Laboratory staff for assistance in setting up the experiment.

Our special thanks to Dr. R. Thun for valuable comments and assistance in data taking.

[1] A. Böhm et al., Phys. Lett. 49B (1974) 491.

[2] E. Nagy et al., Physics lett. 57B (1975.

[3] R.A. Carrigan et al., Phys. Rev. Lett. 24 (1970) 683; J.V. Allaby et al., Nuc. Phys. B52 (1973) 316.

[4] T.T. Wu and C.N. Yang, Phys. Rev. 137B (1965) B708; T.T. Chou and C.N. Yang, Phys. Rev. 170 (1968) 1591; Phys. Rev. Lett. 20 (1968) 1213.

[5] L. Durand and R. Lipes, Phys. Rev. Lett. 20 (1968) 637; M. Elitzur and R.G. Lipes, Phys. Rev. D7 (1973) 1420.

[6] R.J.N. Phillips and V. Barger, Phys. Lett. 46B (1973) 412. [7] V. Bartenev et al., Phys. Rev. Lett. 31 (1973) 1367. 